\title{
White light generating semiconductor nanocrystal luminophors with high photometric quality
}

\author{
Hilmi Volkan Demir \\ Department of Electrical and Electronics Engineering and Department of Physics \\ Bilkent University, Bilkent, Ankara, Turkey TR-06800
}

\begin{abstract}
We proposed and demonstrated warm white light generating combinations of semiconductor nanocrystal quantum dot emitters with high photometric quality including high color rendering index $(-80)$ on LED platforms to meet requirements of future lighting. Additionally, we developed and demonstrated plasmon coupling of these nanocrystal luminophors with metal nanoparticles to control and enhance their spontaneous emission in the solid state film.
\end{abstract}

\section{INTRODUCTION}

Today lighting consumes $20 \%$ of electrical energy production worldwide. Solid state lighting is expected to reduce the global energy demand of lighting by $50 \%$ and consequently reduce the global carbon emission by 300 million tons per year. Presently commercially available white LEDs typically provide cool white light with a low color rendering index $(\sim 70)$. This limits the wide-scale use of these LEDs.

To address this problem, we proposed and demonstrated warm white light generating combinations of semiconductor nanocrystal quantum dot emitters made of CdSe/ZnS coreshell structure $[1,2]$ with high photometric qualities including high color rendering index $(\sim 80)$ on InGaN/GaN LED platforms to meet requirements of future lighting [3].

Also, we developed and demonstrated plasmon engineering of these nanocrystal luminophors coupled with metal nanoparticles (nano-Ag island film) to control and enhance their spontaneous emission in the solid state film [4].

In this presentation, we provide a brief description of our design and characterization of these semiconductor nanocrystal emitters, which hold great promise for use in high-quality lighting.

\section{DESIGN AND CHARACTERIZATION}

Semiconductor nanocrystal emitters that feature relatively narrow band emission (e.g., full width at half maximum $<30$ $\mathrm{nm}$ in solution) allow for obtaining high color rendering index, while keeping the chromaticity operating point within the white region in the chromaticity diagram. This is made possible particularly because of the quantum size effect, which facilitates the precise tuning of peak emission wavelength of these nanocrystal emitters. Thus, designing a right color-converting combination of nanocrystals, it is in principle possible to achieve any emission spectrum as desired. Consequently, our hybrid LEDs integrated with nanocrystal emitters can be carefully fine-tuned to achieve the desired photometric properties

For the optical characterization of our resulting nanocrystal integrated LEDs, we measured the operating chromaticity coordinates, the color rendering index, the correlated color temperature, and the luminous efficacy of optical radiation, as summarized in Table 1.

TABLE I.

TRISTMMULUS COORDINATES, COLOR RENDERING INDEX, CORRELATED COLOR TEMPERATURE, AND

LUMINOUS EFFICACY OF OPTICAL RADIATION.
\begin{tabular}{|l|l|c|}
\hline Figure of merit & Explanation & Unit \\
\hline Tristimulus coordinates $(\mathrm{x}, \mathrm{y})$ & $\begin{array}{l}\text { locus of the perceived color } \\
\text { on the chromaticity diagram }\end{array}$ & - \\
\hline Color rendering index (CRI) & $\begin{array}{l}\text { ability to render true colors } \\
\text { from illuminated objects }\end{array}$ & - \\
\hline $\begin{array}{l}\text { Correlated color temperature } \\
(C C T)\end{array}$ & $\begin{array}{l}\text { temperature of the planckian } \\
\text { blackbody radiator closest in color }\end{array}$ & $\mathrm{K}$ \\
\hline $\begin{array}{l}\text { Luminous efficacy of optical } \\
\text { radiation }(L E)\end{array}$ & $\begin{array}{l}\text { usable radiation for human eye } \\
\text { per optical power }\end{array}$ & $1 \mathrm{~m} / \mathrm{W}$ \\
\hline
\end{tabular}

The operating principle of our hybrid white LEDs integrated with nanocrystal emitters relies on the collective use of the nanocrystals as the luminophor layer and the LED as their optical pump source. When electrically driven, the integrating LED optically excites these nanocrystal luminophors. As a result, the nanocrystal photoluminescence and the LED electroluminescence collectively generate the white light together.

In this work, we used InGaN/GaN LED emitting at 452 $\mathrm{nm}$ as one of primary colors (blue). To develop our hybrid warm white LEDs, we used green- and red-emitting $\mathrm{CdSe} / \mathrm{ZnS}$ core-shell nanocrystals with their emission peaks at 555 and $613 \mathrm{~nm}$, respectively, as the second (green) and third (red) primary colors. These nanocrystals were integrated in the host PMMA matrix on top of the blue LEDs.

To achieve white light generation with warm color temperature and high color rendering index, we analyzed the blackbody radiators on the planckian curve in CIE chromaticity diagram. Based on our analysis, we determined the correct amount of nanocrystals to be integrated on our LED for high performance. Using our careful designs and hybridization of the nanocrystal emitters, we proposed and demonstrated three design sets of proof-of-principle warmwhite LEDs with high-quality white light properties [3].

These led to the photometric properties of 1.) the tristimulus coordinates $(x, y)=(0.37,0.30)$, color rendering index $\mathrm{CRI}=82.4$, correlated color temperature $\mathrm{CCT}=3228 \mathrm{~K}$, and luminous efficacy of optical radiation $\mathrm{LE}=307 \mathrm{~lm} / \mathrm{W} ; 2$.) $(\mathrm{x}, \mathrm{y})=(0.38,0.31), \mathrm{CRI}=81.0, \mathrm{CCT}=3190 \mathrm{~K}$, and $\mathrm{LE}=323$ $\mathrm{lm} / \mathrm{W}$; and 3. $(\mathrm{x}, \mathrm{y})=(0.37,0.30), \mathrm{CRI}=79.6, \mathrm{CCT}=1982 \mathrm{~K}$, 
and $\mathrm{LE}=303 \mathrm{~lm} / \mathrm{W}$; these are also summarized in Table II. The chromaticity coordinates of these implementations are shown in the CIE 1931 chromaticity diagram in Fig. 1 as well [3].

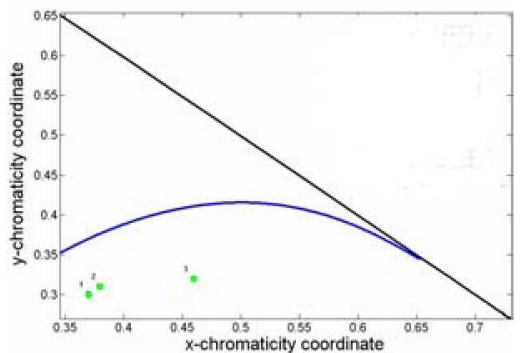

Fig. 1. CIE chromaticity coordinates of our nanocrystal integrated warmwhite light emitting diodes (in green), Sample 1-3, along with the planckian curve of blackbody radiators in the region (in blue).

TABLE II.

OPTICAL PROPERTES OF OUR NANOCRYSTAL INTEGRATED WARM-WHTTE LIGHT EMITTTNG DIODES
\begin{tabular}{|c|c|c|c|c|c|}
\hline Sample & $\mathbf{x}$ & $\mathbf{y}$ & $\mathbf{L E}(\mathbf{m} / \mathrm{W})$ & $\mathrm{CRI}$ & $\mathbf{C C T}(\mathbf{K})$ \\
\hline \hline 1 & 0.37 & 0.30 & 307 & 82.4 & 3228 \\
\hline 2 & 0.38 & 0.31 & 323 & 81.0 & 3190 \\
\hline 3 & 0.46 & 0.32 & 303 & 79.6 & 1982 \\
\hline
\end{tabular}

Using metal nanoisland films, we also developed and demonstrated localized plasmonic resonance coupling of nanocrystal emitters with proximal randomly-distributed metallic nanoislands that are carefully tuned both spectrally and spatially [4]. As a result of the plasmon coupling, we modified the emission linewidth of these nanocrystals, shifted their peak emission wavelength, and enhanced their emission intensity. Using such randomly-distributed Ag nanoparticles, as shown in Fig. 2, we showed controlled modifications of spontaneous emission from CdSe/ZnS nanocrystal emitters, exhibiting such significant emission linewidth and peak modifications, along with high emission intensity enhancement.

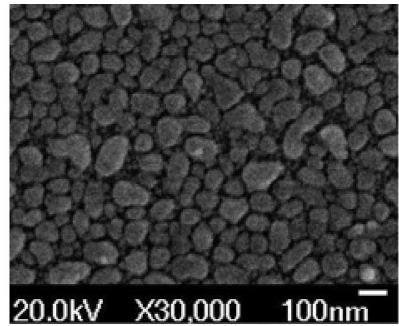

Fig. 2. SEM image of our nano- $\mathrm{Ag}$ island film with $20 \mathrm{~nm}$ mass thickness after it was annealed at $300^{\circ} \mathrm{C}$ for $10 \mathrm{~min}$.

Figure 3 shows collective photoluminescence spectrum from the $\mathrm{CdSe} / \mathrm{ZnS}$ nanocrystal emitters in the vicinity of nano- $\mathrm{Ag}$ film $\left(20 \mathrm{~nm}\right.$ thick, annealed at $300^{\circ} \mathrm{C}$ for $10 \mathrm{~min}$ ) on the bottom, separated by a $10 \mathrm{~nm}$ thick silicon oxide thin film between them. Here this sample was excited at $325 \mathrm{~nm}$ using a He-Cd laser at room temperature. In Fig. 3, this emission spectrum is depicted along with those of the two control groups, one that contains the same CdSe/ZnS nanocrystals alone and another that contains the same CdSe/ZnS nanocrystals with identical nano-Ag $(20 \mathrm{~nm})$ but no dielectric spacer.

In these photoluminescence spectra, we observed that the emission linewidth of the $\mathrm{CdSe} / \mathrm{ZnS}$ core-shell nanocrystals was narrowed down by $10 \mathrm{~nm}$, corresponding to more than $22 \%$ reduction of their full width at half maximum. Furthermore, their peak emission wavelength was substantially shifted by $14 \mathrm{~nm}$. Finally, their photoluminescence intensity was significantly enhanced by 15.1 and 21.6 times on the average compared to the two control groups of the same nanocrystals without $\mathrm{Ag}$ nanoislands (when with no plasmonic resonance) and the same nanocrystals with identical $\mathrm{Ag}$ nanoislands but no dielectric spacer (when quenched by $70 \%$ ), respectively. In all of these characterizations, the same amounts of nanocrystals were used and their photoluminescence measurements were all taken under identical conditions.

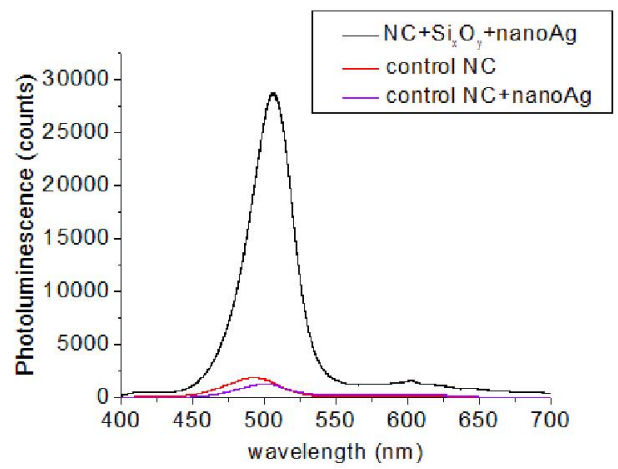

Fig. 3. Photolumineseence spectrum of $\mathrm{CdSe} / \mathrm{ZnS}$ nanocrystals (NC) with nano-Ag ( $20 \mathrm{~nm}$ thick) and a dielectric spacer $(10 \mathrm{~nm}$ thick silicon oxide) between them, compared with those of the control groups that contain the same CdSe/ZnS NCs alone and the same CdSe/ZnS NCs with identical nano- $\mathrm{Ag}$ ( $20 \mathrm{~nm}$ thick) but no dielectric spacer.

\section{CONCLUSIONS}

We presented warm-white light emitting diodes integrated with semiconductor nanocrystal quantum dot emitters to achieve high color rendering index. In this work using nanocrystal emitters in the right color-converting combination enabled us to obtain highly warm correlated color temperature, while keeping their operating chromaticity coordinates in the white region and sustaining their high color rendering index. Also, using randomlydistributed metal nanoparticles, we demonstrated plasmon coupling of these nanocrystal emitters in their proximity to control and enhance their spontaneous emission in the solid state film.

\section{ACKNOWLEDGMENT}

This work is supported by ESF EURYI, EU PHOREMOST NoE 511616, EU MC IRG MOON 021391, TUBITAK (EEEAG 106E020, 104E114, 107E088, 107E297, 105E065 105E066), DPT UNAM, and TUBA GEBIP.

\section{REFERENCES}

[1] S. Nizamoglu, T. Ozel, E. Sari, and H. V. Demir, Nanotechnology, vol. 18 , no. 6, pp. 065709 (2007).

[2] S. Nizamoglu and H. V. Demir, Nanotechnology, vol. 18, no. 40, pp. 
405702 (2007).

[3] S. Nizamoglu, G. Zengin, and H. V. Demir, Applied Physics Letters, vol.

$$
92 \text {, pp. } 031102 \text { (2008). }
$$

[4] I. M. Soganci, S. Nizamoglu, E. Mutlugun, O. Akin, and H. V. Demir, Optics Express, vol. 15, no. 22, pp. 14289-14298 (2007). 